\title{
NFC TECHNOLOGY IN PUBLIC TRANSPORT
}

This article discusses the possibility of the application of the NFC technology as a system of selling tickets in public transport. The NFC (Near Field Communication) technology is a wireless and contactless technology of transmission of radio data over short distances (max. $5 \mathrm{~cm}$ ).

Keywords: NFC technology, public transport.

\section{Introduction}

Public transport operators and authorities, financial service providers, telecommunications operators need to work together to combine their products on a single card. The most promising are smart cards and mobile ticketing. At present smart cards (making use of Radio Frequency Identification [RFID] technology) are still the most common form of e-ticketing. It is likely that, in the future, ticketing applications will evolve to be integrated into bank cards and/or Near Field Communication (NFC) enabled smartphones.

\section{NFC technology}

The NFC technology is a wireless and contactless technology of transmission of radio data over short distances (max. 5 $\mathrm{cm}$ ), enabling an easy and secure either-way communication. This technology is very similar to the RFID (Radio Frequency Identification) technology.

The use of this technology in practice would mean a significant change in the quality of passenger transport. It is possible to integrate payment or loyalty cards, IDs and driving licenses, electronic keys or travel documents into the NFC technology. The use of the NFC technology has many advantages which result from noncash payments and the new advantages include:

- in case of the card expiry date it is not necessary to visit a bank institute to get a new card,

- the NFC technology can also replace physical or virtual keys (car, home, log into the PC),

- the NFC technology facilitates communication between all devices, which are currently widely used.
In order to use the passive NFC technology it is not necessary to own a mobile phone supporting this technology, it is enough to have the passive NFC chip (a so-called sticker), powered by the electromagnetic field of a payment terminal. Stickers take the form of a sticky label. In order to actively use the NFC technology, it is necessary to own a mobile phone, especially a smartphone that is equipped with an NFC chip. The most important brands of mobile phones supporting the NFC as of April 2015 include phone brands:

- Samsung 28 mobile devices,

- Sony 16 mobile devices,

- Apple 14 mobile devices,

- Nokia 13 mobile devices,

- further brands include LG, Xiaomi, Huawei etc.

Technical data and parameters of the NFC:

- the NFC technology has a reserved bandwidth and operates at a frequency of $13.56 \mathrm{MHz}$,

- the data transfer rate is 106,212 or $424 \mathrm{kbps}$,

- the intended speed is $1 \mathrm{Mbps}$,

- the NFC technology uses radio waves for transfer,

- the transmission is Half-Duplex,

- the NFC connects two modes - active and passive,

- it uses its own Open Source protocol NFC.

The NFC technology has a wide range of applications, especially in mobile phones. In this case, via this technology the user can pay, buy tickets or exchange contacts with other devices supporting this technology [1,2 and 3]. Table 1 shows the summary of different wireless technology comparative details.

\footnotetext{
* ' Eva Brumercikova, ${ }^{1}$ Bibiana Bukova, ${ }^{2}$ Leszek Krzywonos

${ }^{1}$ Department of Railway Transport, Faculty of Operation and Economics of Transport and Communications, University of Zilina, Slovakia

${ }^{2}$ Politechnika Lubelska, Lublin, Poland

E-mail: eva.brumercikova@fpedas.uniza.sk
} 
Summary of the comparative details [4]

Table 1

\begin{tabular}{|l|c|c|c|c|c|}
\hline \multicolumn{1}{|c|}{ Concept } & NFC & Bluetooth & WIFI & GPRS/3G & $\begin{array}{c}\text { NFC observation } \\
\text { for public } \\
\text { transport }\end{array}$ \\
\hline Transaction distance & $0.1 \mathrm{~m}$ & $10-100 \mathrm{~m}$ & $\begin{array}{c}300 \mathrm{~m}- \\
40 \mathrm{~km}\end{array}$ & $1 \mathrm{~km}-35 \mathrm{~km}$ & $\begin{array}{c}\text { Intended for a } \\
\text { voluntary action }\end{array}$ \\
\hline Transmission speed & $\mathbf{8 4 8 \mathrm { kbit } / \mathrm { s }}$ & $2 \mathrm{Mbit} / \mathrm{s}$ & $54 \mathrm{mbit} / \mathrm{s}$ & $14 \mathrm{Mbit} / \mathrm{s}$ & Acceptable speed \\
\hline Connection set up time & 20 milliseconds & $6 \mathrm{~s}$ & $2 \mathrm{~s}$ & $1 \mathrm{~s}$ & Extremely fast \\
\hline Connection reliability & Extremely high & Low & Medium & Medium & Extremely reliable \\
\hline Robustness against interferences & Extremely high & Normal & Normal & Normal & Extremely robust \\
\hline $\begin{array}{l}\text { Compatibility with transport } \\
\text { infrastructures }\end{array}$ & Almost total & Zero & Zero & Zero & $\begin{array}{c}\text { Compatibility with } \\
\text { infrastructure }\end{array}$ \\
\hline Compliance with transaction times & Total & No & No & No & $\begin{array}{l}\text { Acceptable } \\
\text { transaction times }\end{array}$ \\
\hline
\end{tabular}

\section{Mobile NFC in transport today}

The usage of mobile NFC transport-related services depends on consumers having NFC handsets that are compatible with the ISO/IEC 14443 standard that forms the basis for contactless infrastructure in the transport industry. These handsets should be certified to ensure that they comply with the full NFC specification, but industry-wide certification mechanisms don't yet exist. The availability of NFC handsets is now growing quickly. By 2015, 50\% of the smartphones shipped will support NFC, according to one leading semiconductor vendor. By then, the vast majority of handsets sold in Europe will likely be smartphones.

Mobile NFC services have already been deployed commercially in several countries, such as Japan, South Korea, France and Turkey.

\section{Preparations for rollout in European Union countries}

Several of Europe's transport operators are adapting their existing electronic ticket systems to support NFC handsets. In this section, we outline some examples [5]:

\section{Deutsche Bahn}

Deutsche Bahn, Germany's main train operator, is preparing to expand its NFC support for the Touch\&Travel system. Touch\&Travel is designed to collect fares automatically by enabling passengers to check in and out of the public transport system using touchpoints. Today, the passenger checks in and out by entering the touchpoint ID number into the Touch\&Travel app on their handset or scanning a 2D barcode. Alternatively, a customer can ask the app to find their location (using technologies, such as GPS and/or Wi-Fi) and then choose from a list of local stations. The handset then transmits the station information over the mobile network to Deutsche Bahn's systems, which charges the journey to the passenger's postpaid account, which is settled once a month. Following an extensive pilot phase, Deutsche Bahn is now preparing to enable passengers to touch their Android and Symbian handset against an NFC tag to check-in and check-out of the transport system.

\section{Transport for London}

Transport for London is planning to gradually replace its proprietary Oyster electronic ticketing system with a system that simply charges a passenger's debit or credit card for each journey made. Such a system will eventually enable Transport for London to reduce the cost of fare collection, as passengers will no longer need to purchase a dedicated plastic card and load money onto that card. Instead of tapping their Oyster card against a reader, the passenger will be able to tap a contactless debit or credit card compatible with the EMV standard. If a debit or credit card is stored in the passenger's NFC handset, then they will be able to use their phone in the same way.

Transport for London is developing software that will register each time the passenger taps in and out of the system. At the end of the day, the software will tally up the journeys made and charge the passenger's payment card via the existing EMV system. The passenger's daily expenditure will be capped to ensure that they aren't charged more than the cost of a day travelcard.

Transport for London is talking to the UK's mobile operators about enabling their wallets to track the passenger's spending on travel in London in real time. That would enable the passengers to 
see how much they have spent during the day, rather than waiting for the settlement to take place at the end of the day.

Transport for London intends to also introduce its own TfL card for people who don't have an EMV card or would prefer to use a prepaid system, in which case they buy credit up front, to control their spending. Again a passenger could store a virtual version of this card in an NFC handset.

\section{Barcelona ATM, Spain}

Barcelona ATM is the governing body for integrated fare collection covering the metro system, railways, tram systems, buses, and other forms of public transport within Barcelona and the 250 towns in the surrounding area. The agency provides the system for approximately 74 transport operators in the Barcelona metropolitan region, which covers 3,240 square kilometers and serves more than five million residents. More than $70 \%$ of the journeys made by customers are with integrated tickets.

ATM is now deploying a full NFC-contactless solution for ticketing, based on open standards and ISO-CEN compliant, which is intended to be a significant step towards the deployment of contactless services across the rest of Catalonia, as well as interoperability with transport systems in other regions. ATM is also building on the momentum of Barcelona City Council's TAP@GO project, which is enabling NFC-payments and municipal services within the city.

\section{Development of the number of payment cards in Slovakia}

Currently, payment cards have become a modern means of payment. It is a comfortable and safe tool for noncash bank transfers of financial resources. A payment card allows its holder to pay for goods in shops, restaurants, hotels, petrol stations or on the internet. Public transport is one possibility to widen the range of the use of payment cards. In the public transport cash payments and noncash payments are currently used only if purchasing tickets over the internet or payments with payment cards when purchasing tickets at the ticket office. The following Table 2 shows the development of the number of payment cards. The source of the presented data is the Slovak Banking Association [6]. This statistics provides relevant data about the number of payment cards issued in the Slovak Republic as well as the volume of transactions made with payment cards.

The given table demonstrates an increase in the number of payment cards as well as the volume of transactions made by payment cards. For comparison, the volume of transactions made in 2013 compared to 2008 increased by $145 \%$. The number of payment cards issued increased by $158 \%$.

In 2015, a survey of the interest and knowledge of public passengers about the NFC technology was conducted. The research involved 504 respondents who were contacted either directly or through the website on which a questionnaire was published. The research surveyed the knowledge of passengers about the NFC technology. The assumption was that the passengers will not have knowledge about this technology. It is a new platform through which payments can be made. The research results confirmed the low awareness of the respondents about the NFC technology. Almost $60 \%$ of respondents said they do not know about this technology. Interestingly, respondents with primary education knew the most about the NFC technology (63.64\%). Subsequently, the respondent was informed about the NFC technology. After explaining what the NFC technology means, the respondents were asked whether they would use this technology when buying a ticket. Most respondents answered positively (37.09\%), "do not know" said $25 \%$ of respondents, and certainly not $35.91 \%$. Respondents who answered "no" explained that the reason was their concern particularly in case their mobile phone would be stolen and they were also worried about what to do in case the phone would turn off because of a discharged battery.

Development of the number and volume of card transactions in Slovakia [Authors]

Table 2

\begin{tabular}{|c|c|c|c|c|c|c|c|c|}
\hline Together & $\begin{array}{l}\text { Number of } \\
\text { prepaid } \\
\text { cards }\end{array}$ & $\begin{array}{l}\text { Value of } \\
\text { trans actions } \\
\text { by prepaid } \\
\text { cards (in } \\
\text { EUR) } \\
\end{array}$ & $\begin{array}{l}\text { Number of } \\
\text { debit cards }\end{array}$ & $\begin{array}{l}\text { Value of } \\
\text { trans actions - } \\
\text { by de bit } \\
\text { cards (in } \\
\text { EUR) }\end{array}$ & $\begin{array}{l}\text { Number of } \\
\text { cre dit cards }\end{array}$ & $\begin{array}{l}\text { Value of } \\
\text { trans actions } \\
\text { by cre dit } \\
\text { cards (in } \\
\text { EUR) } \\
\end{array}$ & $\begin{array}{l}\text { Number of } \\
\text { contactless } \\
\text { cards }\end{array}$ & $\begin{array}{l}\text { Value of } \\
\text { trans actions } \\
\text { by } \\
\text { contactless } \\
\text { cards (in } \\
\text { EUR) } \\
\end{array}$ \\
\hline 31.12 .2008 & - & - & 3913009 & 3208859621 & 1321382 & 197467398 & - & - \\
\hline 31.12 .2009 & - & - & 3998137 & 3273197274 & 1062065 & 177557824 & - & - \\
\hline 31.12 .2010 & - & - & 4176196 & $3648 \quad 132517$ & 795777 & 176247505 & - & - \\
\hline 31.12.2011 & 9648 & 593645 & 4520612 & 3977112088 & 818132 & 191444893 & 392387 & - \\
\hline 31.12 .2012 & 51587 & 601000 & 3929979 & 4232266519 & 604992 & 199587443 & 1207052 & 15646327 \\
\hline 31.12 .2013 & 50309 & 659915 & 4115198 & 4450199795 & 620776 & 223522290 & 2150984 & 100932358 \\
\hline 31.12 .2014 & 70175 & 13704482 & 4313089 & 4892084535 & 654281 & 247927042 & 3216030 & 229471014 \\
\hline
\end{tabular}




\section{Cycle product - NFC in mobile phone}

This chapter describes the processes in which a transport card is involved when it is in an NFC mobile phone. The purpose is to give a view of the card life cycle and the implications involved for successfully achieving the implementation of the NFC mobile phone in public transport.

\subsection{Differences when the card is in the NFC Mobile Phone}

When a transport card is placed in a mobile phone, some important differences occur that need to be pointed out for a better understanding of its implementation implications in public transport.

- The transport card becomes "virtualized" inside the mobile phone's secure element (SE), therefore ceasing to exist as a physical card or physical chip.

- The transport ticket is also "virtualized" inside the card, but this in fact is nothing new as it is already happening in current transport cards.

- The SE is a support that will be able to hold other transport cards or other types such as bank cards, as well as other services, communications etc., which means that it can no longer be owned by the transport company.

- Since the card will be located inside the mobile phone, it may be accessed remotely at any time communication is activated. The mobile phone environment, and more with the NFC environment, makes it possible to make payment transactions from the mobile phone, which leads the way to card top-ups without having to go to a top-up point.

- As NFC technology is currently an emerging technology and has many requirements associated, it cannot always be guaranteed that the card will be downloadable to all mobile phone models in the market, meaning that the user will always have a choice during card request process on the mobile phone. The contactless card has certain limitations, among them its interaction capacity: we can only obtain information on the card if we hold it near a terminal that powers it and is able to authenticate and obtain the corresponding information. NFC makes it possible to remove this obstacle, since mobile phones today have large screens, connectivity and processing capability [7].

\subsection{Ticket life cycle}

The ticket life cycle diagram is complex. By way of example, a typical diagram is included to show it in Fig. 1.

Identity control in the case of personalized tickets - if the ticket requires the user to have specific characteristics. The user must be clearly identified despite not having a physical card with a photograph.

Transactions in general by lists - contactless cards are processed remotely by lists of cards that are sent to the devices and when they locate them, they act. With NFC technology, this may be done immediately. For example, if the user has 3 additional trips due to an incident, this balance may be increased remotely at any time.

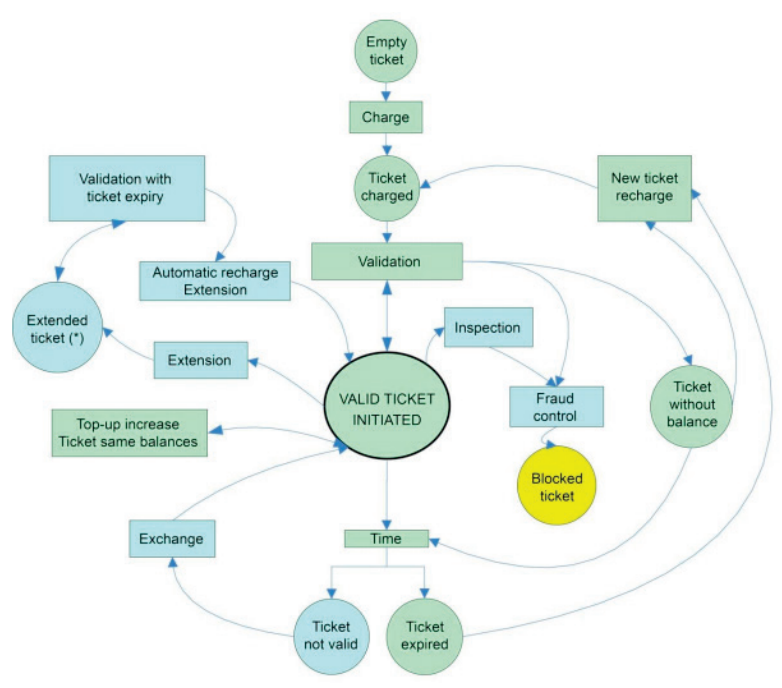

Fig. 1 Ticket life cycle [7]

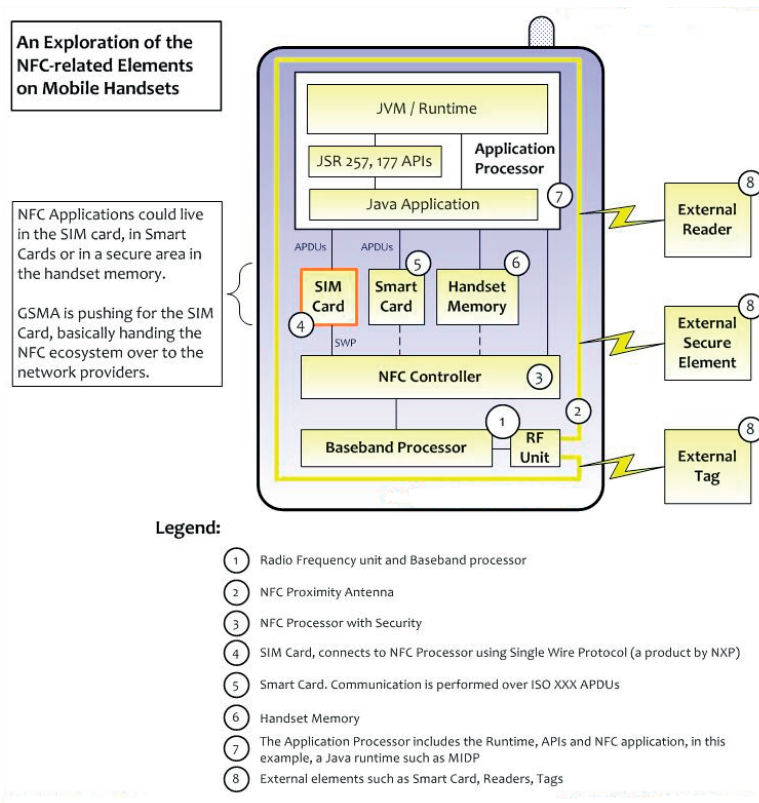

Fig. 2 An exploration of the NFC - related Element on mobile handsets [8]

Remote Charging - the ticket may be recharged remotely and in real time, without the need to have to wait to pass a top-up or 
validation point which has been reached by a list of cards that have made a top-up purchase on the web site [7].

The following diagram (Fig. 2) shows An Exploration of the NFC-related elements on mobile handsets.

\section{Possibilities of NFC structural simulation}

Near field communication (NFC) technology is attracting a lot of interest for mobile payment and ticketing applications. Behind the simple "swipe and go" operation is a complex system spanning many different standards and presenting challenges from the RF interface to the digital processing. Broadcom is currently developing chips to provide NFC solutions for handset manufacturers, and MATLAB and Simulink are an important part of both the system modelling and, through HDL Coder, generation of the actual digital hardware [9].

The internal structure of the NFC technology is shown in Fig. 3. This NFC model uses only a standard ASK modulation scheme and includes also an AGC support.

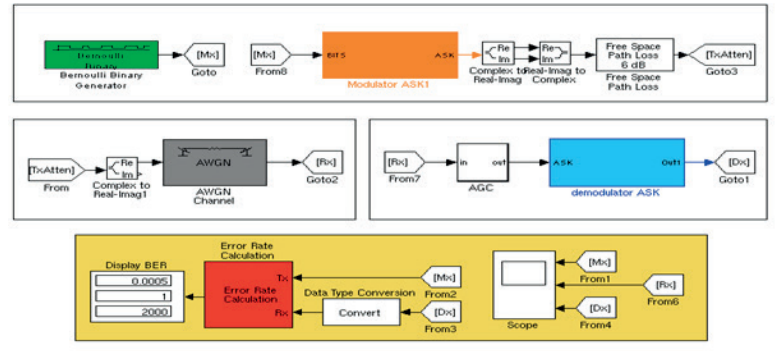

Fig. 3 The internal structure of the NFC technology [9]

\section{Conclusion}

Most experts agree that NFC is the smart technology of the future and that phone-based applications will become increasingly important. NFC applications can integrate several independent services and hold the promise of freeing single operators from the need to set up a cost-intensive infrastructure of check-in/check-out devices. Instead, the users - with their mobile phones or with their contactless bank cards - provide a large portion of the necessary infrastructure. Modern contactless (be-in/be-out) technologies in public transport, for example, do not even require the passenger to actively register at the ticket gate. Instead, a tag at each station or in each vehicle automatically detects and registers new passengers when they enter, during the trip and at the end to automatically calculate the most favorable fare. However, experts agree that customers want to have a choice among different payment options and that phone-based applications will not appeal to everyone. Therefore, different payment options should remain available. At present and in the medium term, smart cards offer an attractive medium to connect payment options in public transport with additional services - however, they require operators for larger investments in access control infrastructures. In either case, a change in the ticketing environment must be accompanied by a communication strategy explaining the change in detail, and emphasizing its benefits for all actors that are involved in the process [10 and 11].

\section{Acknowledgment}

This article was created to support the project named as:

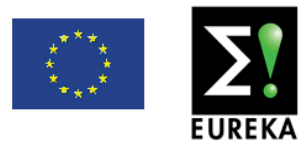

E!7619 TABLOG - Use of mobile devices such as tablets\&smartphones for data collection, data processing \& operational process management.

\section{References}

[1] BRUMERCIK, F., TOMASIKOVA, M., NIEOCZYM, A.: Epicyclic Gear Train Synthesis, Communications - Scientific Letters of the University of Zilina. No. 3, 2015, pp. 47-50, ISSN 1335-4205.

[2] BRUMERCIK, F, DANKO, R.: Transport Application of Hybrid Simulation, Communications - Scientific Letters of the University of Zilina. No. 2, 2014, pp. 20-24, ISSN 1335-4205.

[3] TRPISOVSKY, M., PRUSA, P.: Regional Public Transportation Services Modelling, Nase more: J. of Marine Science, 2014, vol. 61, No. 3-4, pp. 77-82, ISSN 0469-6255

[4] PUHE, M., EDELMANN, M., REICHENBACH, M.: Integrated Urban e-ticketing for Public Transport and Touristic Site, Science and Technology Options Assessment. Brussels, 2014, ISBN 978-92-823-5302-8.

[5] http://www.gsma.com (16. 10.2015)

[6] http://www.sbaonline.sk/sk/bankovy-sektor/statistika (16. 10.2015)

[7] INTELLIGENT TRANSPORT SYSTEM. White paper of the application of public transport, 2013, ISBN 978-84-616-4714-9. 
[8] http://weblog.cenriqueortiz.com/touch-nfc/ (16. 10.2015)

[9] http://www.mathworks.com (16. 10.2015)

[10] KENDRA, M.: Integration of Individual Car Transport and Public Passenger Transport in Cities, OPTi: $1^{\text {st }}$ intern. conference on Engineering and applied sciences optimization, 2014, ISBN 978-960-99994-6-5.

[11] KAMPF, R., LIZBETIN, J., LIZBETINOVA, L.: Requirements of a Transport System user, Communications - Scientific Letters of the University of Zilina. No. 4, 2012, pp. 106-112, ISSN 1335-4205. 